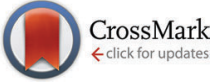

Cite this: Phys. Chem. Chem. Phys., 2016, 18, 25637

Received 23rd June 2016 Accepted 16th August 2016

DOI: $10.1039 / c 6 c p 04398 d$

www.rsc.org/pccp

\section{Photoinduced water splitting in pyridine water clusters $\uparrow$}

\author{
Natalia Esteves-López, Stephane Coussan, Claude Dedonder-Lardeux and \\ Christophe Jouvet*
}

\begin{abstract}
Ab initio calculations predict that pyridine (Py) can act as a photo-catalyst to split water by the absorption of a UV photon following the reaction $\mathrm{Py}-\mathrm{H}_{2} \mathrm{O}+h \nu \rightarrow \mathrm{PyH}^{\bullet}+\mathrm{OH}^{\bullet}$. To test this prediction, we performed two types of experiments: in the first, we characterize the electronic spectroscopy of the $\mathrm{PyH}^{\bullet}$ radical in the gas phase. In the second, we evidence the reaction through the UV excitation of molecular $\mathrm{Py}-\left(\mathrm{H}_{2} \mathrm{O}\right)_{n}$ clusters obtained in a supersonic expansion and monitoring the $\mathrm{PyH}^{\bullet}$ reaction product. The results show unambiguously that $\mathrm{PyH}^{\bullet}$ is produced, and thus that water is split using pyridine as a photo-catalyst.
\end{abstract}

In this paper we show that pyridine can act as a photo-catalytic molecular system, which can dissociate the water covalent bond with UV C light. A water molecule is a ubiquitous system to produce $\mathrm{H}_{2}$, however VUV light is needed to photo-dissociate the $\mathrm{H}-\mathrm{OH}$ covalent bond $(186 \mathrm{~nm}, 6.66 \mathrm{eV})$. Since most of the sunlight reaching the earth is in the visible spectral region, we cannot dissociate the $\mathrm{H}-\mathrm{OH}$ bond under normal sunlight conditions and it is necessary to design a system that could break the $\mathrm{H}-\mathrm{OH}$ bond with visible light.

Recent theoretical calculations ${ }^{1-3}$ show that, when the mixed pyridine water complex $\left(\mathrm{Py}-\mathrm{H}_{2} \mathrm{O}\right)$ is excited to its first optically active state $\left({ }^{1} \pi \pi^{*}, \sim 4.8 \mathrm{eV}\right)$, water splitting occurs through the following reaction, $\mathrm{Py}-\mathrm{H}_{2} \mathrm{O}+h \nu \rightarrow \mathrm{PyH}^{\bullet}+\mathrm{OH}^{\bullet}$, generating pyridinyl and hydroxyl radicals. The energy for the UV excitation of $\mathrm{Py}-\mathrm{H}_{2} \mathrm{O}$ is $1.8 \mathrm{eV}$ lower than the energy necessary to excite and photo-dissociate water directly. Liu et al. further reported that similar water splitting occurs in the triplet state $\left(\mathrm{T}_{1}\right)$ of $\mathrm{Py}-\mathrm{H}_{2} \mathrm{O}{ }^{3}$ Thus, pyridine acts as a photo-catalyst but to be useful the photo-catalyst should be recovered by the absorption of a second photon. Indeed, the calculations also indicate that the pyridinyl radical can release the additional hydrogen atom when irradiated with visible light $(\sim 600 \mathrm{~nm}, \sim 2 \mathrm{eV})$.

CNRS, Aix-Marseille Université, Physique des Interactions Ioniques et Moléculaires (PIIM) UMR-7345, Marseille, France. E-mail: Christophe.jouvet@univ-amu.fr

$\dagger$ Electronic supplementary information (ESI) available: Table S1: calculated vertical transition energies for $\mathrm{PyH}^{\bullet}$ in $C_{2 \mathrm{v}}$ symmetry. Fig. S1: action spectra of $\mathrm{Py}-\mathrm{H}_{2} \mathrm{O}$, the pump laser is scanned and the detection is set on the $\mathrm{Py}-\mathrm{H}_{2} \mathrm{O}^{+}$mass (lower trace), or on the $\mathrm{PyH}^{+}$mass with the probe laser at $225 \mathrm{~nm}$ (upper trace). Fig. S2: mass spectra obtained for pyridine without water through a one-color twophoton excitation/ionization scheme. Fig. S3: comparison between mass spectra obtained with the pump/probe scheme in an expansion with and without water. Fig. S4: excitation/action spectra of the species in the jet: homodimer, $\mathrm{Py}-\mathrm{H}_{2} \mathrm{O}$ and Py one-color two-photon ionization spectra and pump/probe $(225 \mathrm{~nm}) \mathrm{PyH}^{\bullet}$ and $\mathrm{dPyD}^{\bullet}$ action spectra. See DOI: 10.1039/c6cp04398d
The pyridinyl radicals obtained by the irradiation of pyridine in water have been postulated in the homogeneous reduction of $\mathrm{CO}_{2}$ by pyridine. ${ }^{4}$ The ground state of the $\mathrm{PyH}^{\bullet}-\mathrm{OH}^{\bullet}$ diradical structure has been calculated to be the ion pair structure $\mathrm{PyH}^{+}$ $\mathrm{OH}^{-}{ }^{1}$ Recently, water splitting has been observed in negatively charged $\left[\mathrm{Py}-\left(\mathrm{H}_{2} \mathrm{O}\right)_{n}\right]^{-}$clusters. ${ }^{5,6}$

Molecular clusters have been extensively used to evidence the primary processes involved in excited states of molecules in a well-defined environment. These studies have allowed to evidence very clearly some photochemical reactions such as proton transfer through molecular wires,${ }^{7-10}$ hydrogen transfer to solvents in mixed molecule-solvent ${ }_{n}$ clusters, ${ }^{11-23}$ solvent isomerization, ${ }^{24-27}$ characterization of intermediate states of reactions ${ }^{28-31}$ and chiral discrimination. ${ }^{32}$ The case of pyridinewater clusters is peculiar since some attempts have been made to characterize their spectroscopy but without success. ${ }^{33}$ This is already good indication that some fast non-radiative processes occur, which prevent the detection of $\mathrm{Py}-\mathrm{H}_{2} \mathrm{O}$ clusters through multiphoton ionization or laser induced fluorescence (LIF).

To demonstrate directly the photochemical reaction in Py- $\mathrm{H}_{2} \mathrm{O}$ clusters, and compare with ab initio calculations, the experiment has been conducted in the gas phase, using molecular clusters of pyridine with water produced in a supersonic expansion. After excitation to the ${ }^{1} \pi \pi^{*}$ state of pyridine, two reaction products are expected to be produced, the $\mathrm{OH}^{\bullet}$ radical and the hydrogenated pyridine (pyridinyl radical $\mathrm{PyH}^{\bullet}$ ). In the gas phase the radical pair can separate freely. As will be seen, the ionization potential (IP) of the $\mathrm{PyH}^{\bullet}$ radical is calculated to be rather low, which should make its detection quite easy. It should then be easier to detect the $\mathrm{PyH}^{\bullet}$ radical as a reaction product of excited $\mathrm{Py}-\left(\mathrm{H}_{2} \mathrm{O}\right)_{n}$ clusters than to detect $\mathrm{OH}^{\bullet}$ through laser induced fluorescence, which has a low detection efficiency (typically $10^{-3}$ ). The experimental scheme 
used involves the excitation of Py- $\left(\mathrm{H}_{2} \mathrm{O}\right)_{n}$ clusters and the detection of the $\mathrm{PyH}^{\bullet}$ radical through ionization by one or two photons.

Since the $\mathrm{PyH}^{\bullet}$ radical spectroscopy has not yet been studied, the experiment will be split into two parts, the first one being the spectroscopic characterization of the cold $\mathrm{PyH}^{\bullet}$ radical, and the second one the detection of $\mathrm{PyH}^{\bullet}$ as a reaction product after the excitation of the $\mathrm{Py}-\left(\mathrm{H}_{2} \mathrm{O}\right)_{n}$ clusters. Additional calculations are performed to get information on the nature of the excited states of the $\mathrm{PyH}^{\bullet}$ radical and its ionization potential.

\section{Experimental}

The setup that we used is composed of a pulsed supersonic expansion combined with a time of flight mass spectrometer. The setup has been previously described ${ }^{34}$ and is quite standard. The discharge source is the same as used in previous experiments to produce either cold neutral van der Waals species ${ }^{35}$ or more recently protonated molecular ions. ${ }^{36}$ In a few words, a pulsed high voltage discharge located a few $\mathrm{mm}$ after the pulsed nozzle produces $\mathrm{PyH}^{\bullet}$ radicals from a gas mixture of $\mathrm{He} / \mathrm{H}_{2} /$ pyridine. The radicals are cooled down in the expansion and ionized between the extraction plates of the reflectron Time of Flight (TOF) mass spectrometer (Jordan TOF Products, Inc.). Additional experiments were conducted with fully deuterated pyridine (hereafter named dPy).

In the second type of experiments, the discharge is removed and $\mathrm{Py}-\left(\mathrm{H}_{2} \mathrm{O}\right)_{n}$ (or dPy- $\left.\left(\mathrm{D}_{2} \mathrm{O}\right)_{n}\right)$ clusters are produced by expanding a mixture of water and pyridine (at their vapor pressure at room temperature) with He (between 1 and 5 atm). Two laser beams are temporally and spatially synchronized between the extracting plates of the mass spectrometer, the pump laser excites the clusters and the probe laser detects the $\mathrm{PyH}^{\bullet}\left(\right.$ or $\mathrm{dPyD}^{\bullet}$ ) radicals.

The lasers used are YAG pumped nanosecond OPO lasers (EKSPLA model-NT342B), which have a $10 \mathrm{~Hz}$ repetition rate, a resolution (FWHM) of $8 \mathrm{~cm}^{-1}$ and a minimum scanning step of $0.02 \mathrm{~nm}$. The lasers are focused in the middle of the extraction zone of the time of flight mass spectrometer. These lasers can be scanned from visible to UV in three regions: from 410 to $709 \mathrm{~nm}$ (OPO signal), from 296 to $409.9 \mathrm{~nm}$ (frequency mixing) and from 225 to $295.9 \mathrm{~nm}$ (frequency doubling).

Calculations are also performed using the Turbomole package, ${ }^{37}$ at the TD-DFT (B3LYP) and RI-CC2 levels with the aug-cc-pVDZ basis set, to get information on the $\mathrm{PyH}^{\bullet}$ ionization potential and on the excited states of the radical.

\section{Results and discussion}

\section{Spectroscopic investigation of the pyridinyl radical $\left(\mathrm{PyH}^{\bullet}\right)$}

Fig. 1 shows the mass spectra obtained when the probe laser is set at $225 \mathrm{~nm}$ with the discharge on or off. In the absence of discharge (red trace) the ion at $\mathrm{m} / \mathrm{z}=79$ corresponds to the pyridine radical cation $\mathrm{Py}^{+}$produced by the two-photon ionization of pyridine and the small peak at $\mathrm{m} / \mathrm{z}=80$ corresponds to the pyridine isotope with a ${ }^{13} \mathrm{C}$ atom. When the discharge is on,

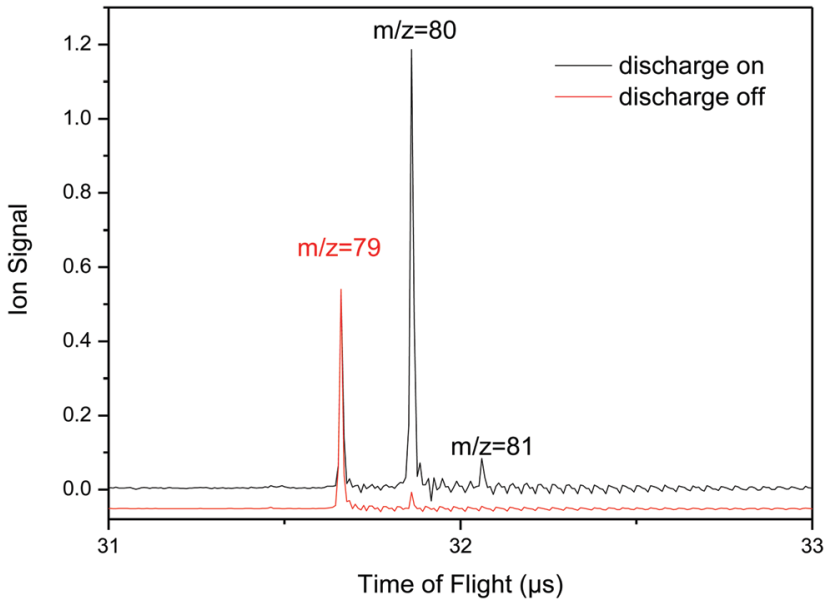

Fig. 1 Mass spectra obtained with a mixture of $\mathrm{He} / \mathrm{H}_{2} /$ pyridine with the discharge source on (black) or off (red), the ionization laser being set at $225 \mathrm{~nm}$.

an intense peak at $m / z=80$ with its isotopic companion at $m / z=81$ is obtained indicating that protonated pyridine $\mathrm{PyH}^{+}$is observed by the ionization of the $\mathrm{PyH}^{\bullet}$ radical.

The spectroscopy of the $\mathrm{PyH}^{\bullet}$ radical is obtained by monitoring the intensity of the $m / z=80$ mass peak $\left(\mathrm{PyH}^{+}\right)$while scanning the laser from visible to UV as displayed in Fig. 2. The onset of the very strong signal at $234 \mathrm{~nm}(5.31 \mathrm{eV})$ and the observation of a continuum at higher energy correspond clearly to the ionization potential of the $\mathrm{PyH}^{\bullet}$ radical. To comfort this assertion, the IP of $\mathrm{PyH}^{\bullet}$ has been calculated using the DFT method with the B3LYP functional and the aug-cc-pVDZ basis

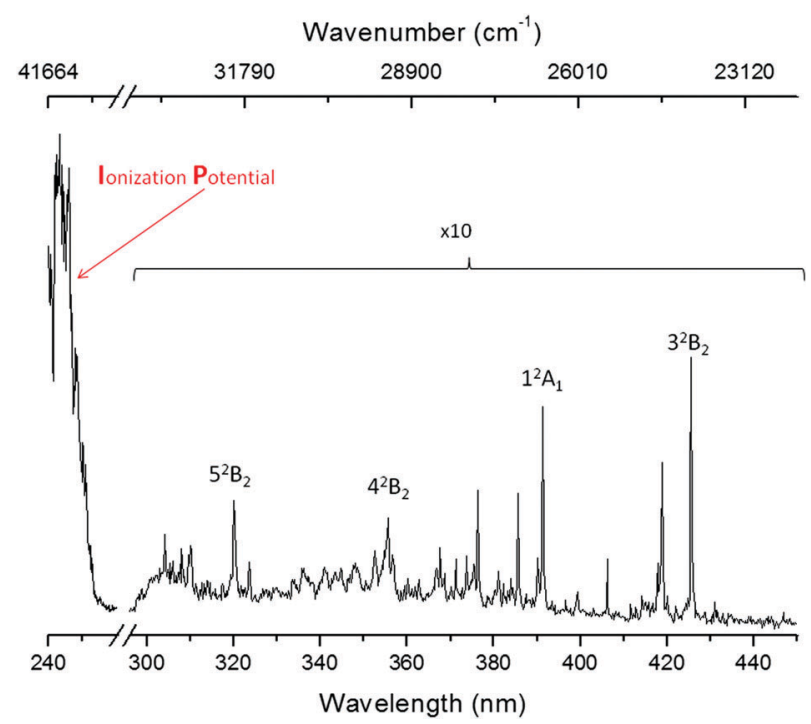

Fig. 2 Spectroscopy of the $\mathrm{PyH}^{\bullet}$ radical produced in the discharge source and cooled down in the supersonic expansion. At high energy the molecule is ionized by one photon. At lower energies, i.e. for excitation wavelengths between 300 and $440 \mathrm{~nm}$, the spectrum is obtained by resonance enhanced one-color two-photon ionization (R2PI). The main bands have been assigned by comparison with ab initio calculations (see the text). 
set (including the difference in zero point energy $\triangle \mathrm{ZPE}$ ), and is found at $235 \mathrm{~nm}(5.28 \mathrm{eV})$.

Some well resolved transitions are observed in the visible and UV A spectral regions. The first band is observed at $425.6 \mathrm{~nm}\left(23496 \mathrm{~cm}^{-1}\right.$ or $\left.2.91 \mathrm{eV}\right)$ and it requires two photons to reach the IP of $\mathrm{PyH}^{\bullet}$. One can detect quite easily the states that can be ionized with two photons i.e. those which have their absorption below $468 \mathrm{~nm}$ (half the IP). Lower energy states would need the absorption of three photons to be detected, and nothing has been observed at energies lower than $425.6 \mathrm{~nm}$. The $\mathrm{dPyD}^{\bullet}$ spectrum shows the same transitions slightly redshifted and is given in Fig. 5c.

The ground state optimized structure has been obtained from calculations at the RI-CC2 (aug-cc-pVDZ) level and shows a $C_{2 \mathrm{v}}$ symmetry. Since the experimental spectrum shows weak Franck Condon activity and strong band origins, the radical should not be too much distorted from this symmetry in the excited states. The excited state optimizations have then been calculated under $C_{2 \mathrm{v}}$ geometry constraints for states which have a significant oscillator strength $\left(>10^{-4}\right)$. The calculated transition energies are systematically higher by $0.3 \mathrm{eV}$ than the experimental transitions (see Table 1). This is the typical uncertainty of CC2 calculations for closed-shell molecules, while the uncertainty for open-shell species cannot be estimated at present due to very few experimental and theoretical data. Based on these calculations, we propose the assignment of Fig. 2. It should be mentioned that the first transition observed corresponds to the fourth excited state and the third state of $B_{2}$ symmetry. We tried to calculate the excited state vibrations but in the absence of symmetry the open shell/CC2 calculation of such a high excited state failed.

It should be mentioned that the $\mathrm{PyH}^{\bullet}$ signal is not very strong because the discharge method is not so efficient to produce the radical, so that the laser is rather powerful and some saturation of the vibronic transitions probably occurs, which may explain why some excited states with a low oscillator strength can be detected. As can be seen in the ESI $\dagger$ (Table S1), there is not so much choice to assign the states and one should keep in mind that open shell excited state calculations may not be so accurate, and that the Franck-Condon factors could not be calculated.

The first two lower excited states have quite similar vibrational spectra. The active vibrations at $380 / 371 \mathrm{~cm}^{-1}$ and $1122 /$ $1005 \mathrm{~cm}^{-1}$ are typical of the active vibrations in pyridine and can be tentatively assigned to the $16 \mathrm{a}^{1}$ and $12^{1}$ modes observed at 323 and $955 \mathrm{~cm}^{-1}$ in the excited state of pyridine and at 314 and $1105 \mathrm{~cm}^{-1}$ in the radical cation. ${ }^{38}$

Table 1 Experimental and calculated transition energies of $\mathrm{PyH}^{\bullet}$

\begin{tabular}{|c|c|c|c|c|}
\hline \multicolumn{2}{|c|}{ Experimental } & \multirow[b]{2}{*}{ State label } & \multirow{2}{*}{$\begin{array}{l}C_{2 \mathrm{v}} \text { optimized } \\
\text { transition }(\mathrm{eV})\end{array}$} & \multirow{2}{*}{$\begin{array}{l}\text { Oscillator } \\
\text { strength }\end{array}$} \\
\hline $\mathrm{nm}$ & $\mathrm{eV}$ & & & \\
\hline 425.6 & 2.91 & $3^{2} \mathrm{~B}_{2}$ & 3.20 & $1 \times 10^{-2}$ \\
\hline 391.2 & 3.17 & $2^{2} \mathrm{~A}_{1}$ & 3.37 & $4 \times 10^{-3}$ \\
\hline 356.0 & 3.48 & $4^{2} \mathrm{~B}_{2}$ & 3.58 & $3 \times 10^{-3}$ \\
\hline 320.0 & 3.88 & $5^{2} \mathrm{~B}_{2}$ & 4.14 & $2 \times 10^{-4}$ \\
\hline
\end{tabular}

Excitation of $\mathrm{Py}-\left(\mathrm{H}_{2} \mathrm{O}\right)_{n}$ or $\mathrm{dPy}-\left(\mathrm{D}_{2} \mathrm{O}\right)_{n}$ clusters: direct evidence of the water splitting reaction

The experiments have been conducted for hydrogenated and fully deuterated species $\mathrm{dPy}$, and the experimental results are basically the same. $\mathrm{Py}-\left(\mathrm{H}_{2} \mathrm{O}\right)_{n}$ clusters produced in the supersonic jet are excited in the energy region of the pyridine ${ }^{1} \pi \pi^{*}$ state (around $255 \mathrm{~nm}$ ) and $\mathrm{PyH}^{\bullet}$ is probed by ionization at $225 \mathrm{~nm}$. The probe laser at $225 \mathrm{~nm}$ ionizes efficiently $\mathrm{PyH}^{\bullet}$ with one photon and excites very weakly the parent clusters (see Fig. S1 in the ESI $\dagger$ ). The delay between lasers is $600 \mathrm{~ns}$, to discriminate $\mathrm{PyH}^{\bullet}$ radicals from the ${ }^{13} \mathrm{C}$ isotope of pyridine (both have the same mass) and to discriminate the reaction in the excited state from a reaction in the ionic state of the clusters due to a two-photon absorption at $255 \mathrm{~nm}$ (the $\mathrm{PyH}^{+}$peak in the upper trace of Fig. 3). Besides, such a delay preludes the absorption of the probe laser from the excited states of the clusters, which have a much shorter lifetime. The ions issued from the ionization of $\mathrm{PyH}^{\bullet}$ by the probe laser are then observed $600 \mathrm{~ns}$ after the ones produced by the pump laser since time $t=0$ for the time of flight is synchronized with the pump laser. The formation of pyridinyl is clearly shown in Fig. 3 in which the signal of $\mathrm{PyH}^{+}$is absent if the pump laser is off and the probe laser is on (middle trace), while when both lasers are present (lower trace) the $\mathrm{PyH}^{+}$ion signal coming from the direct ionization of $\mathrm{PyH}^{\bullet}$ radicals issued from the excitation of $\mathrm{Py}-\left(\mathrm{H}_{2} \mathrm{O}\right)_{n}$ clusters is the most intense peak.

In the upper trace of Fig. 3, the $\mathrm{PyH}^{+}$ion is observed through a one-color two-photon scheme at $255 \mathrm{~nm}$. This signal is not linked to the reaction in clusters with water but to the reaction of pyridine cluster ions. The same experiment has been performed in absence of water in the supersonic expansion. To ensure that there is no water in the tubing bringing the $\mathrm{He} / \mathrm{Py}$

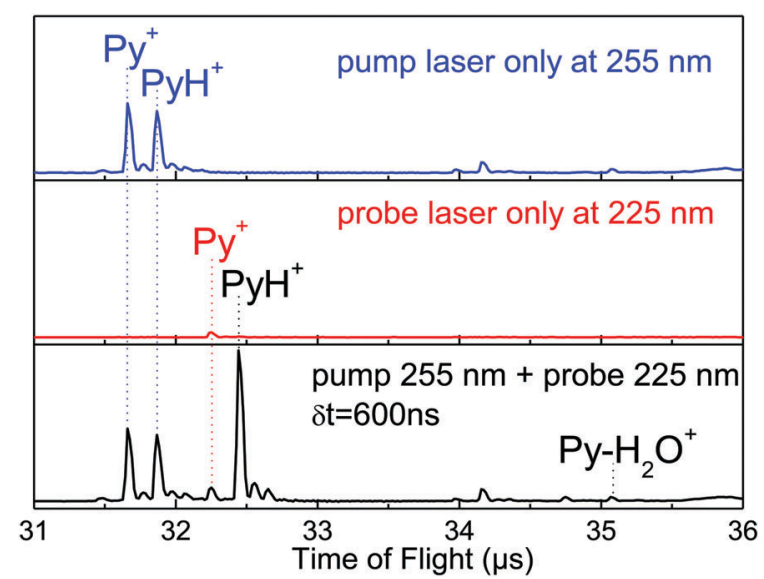

Fig. 3 Mass spectra recorded after excitation of the $\mathrm{Py}-\left(\mathrm{H}_{2} \mathrm{O}\right)_{n}$ clusters. Upper trace: pump laser at $255 \mathrm{~nm}$ alone: the $\mathrm{Py}-\left(\mathrm{H}_{2} \mathrm{O}\right)_{n}$ clusters are excited and the $\mathrm{PyH}^{+}$ion is obtained through one-color two-photon absorption. Middle trace: probe laser at $225 \mathrm{~nm}$ alone: nearly no ion signal since Py and $\mathrm{Py}-\left(\mathrm{H}_{2} \mathrm{O}\right)_{n}$ clusters absorb very weakly at this wavelength. Lower trace: pump $(255 \mathrm{~nm})$ and probe $(225 \mathrm{~nm})$ lasers with the probe laser delayed by 600 ns: the most intense peak is the $\mathrm{PyH}^{+}$ion produced by excitation of the clusters and ionization of the $\mathrm{PyH}^{\bullet}$ radical via a one-photon process. 
gas to the pulsed valve, some desiccant $\left(\mathrm{CaCl}_{2}\right)$ has been introduced in the line, and absolutely no water complex could be obtained. Under these conditions, the signal corresponding to the ionization of the $\mathrm{PyH}^{\bullet}$ radical by the probe laser is negligible, whereas the $\mathrm{PyH}^{+}$signal coming from the reaction in $(\mathrm{Py})_{n}{ }^{+}$clusters $(n=2$ or more) in their ionic state can still be observed (Fig. S2, ESI $\dagger$ ). The comparison between pump/probe mass spectra observed in the presence and absence of water clusters is shown in Fig. S3 (ESI $\dagger$ ).

The spectral signatures of both processes are totally different (see Fig. 4). The $\mathrm{PyH}^{+}$signal issued from the ionic reaction obtained in a water free expansion exhibits a smooth increase going from $225 \mathrm{~nm}$ up to $245 \mathrm{~nm}$ (Fig. 4b). When water is added in the expansion, the excitation of $\mathrm{Py}-\left(\mathrm{H}_{2} \mathrm{O}\right)_{n}$ clusters leads to the formation of a pyridinyl radical, which displays a step function type spectrum, no signal between 245 and $234 \mathrm{~nm}$ and a strong signal between 234 and $225 \mathrm{~nm}$, as observed when
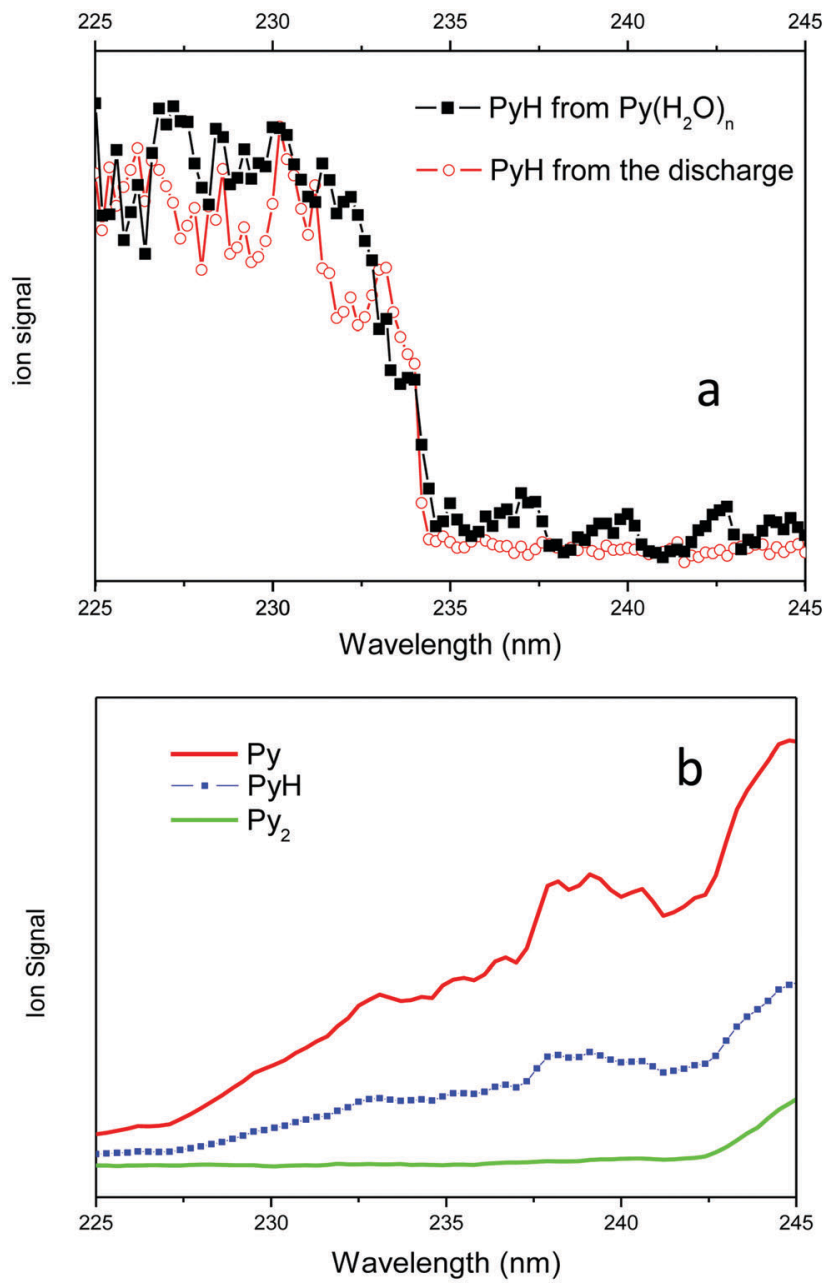

Fig. 4 (a) $\mathrm{PyH}^{+}$signal coming from the ionization of the pyridinyl radical: in red pyridinyl radicals produced by the discharge source; in black pyridinyl radicals obtained from the reaction of pyridine-water clusters excited at $255 \mathrm{~nm}$ and probed by a second laser. (b) $\mathrm{Py}^{+}, \mathrm{PyH}^{+}$and $\mathrm{Py}_{2}{ }^{+}$ signals obtained from multiphoton ionization of $\mathrm{Py}, \mathrm{PyH}^{\bullet}$ and $\mathrm{Py}_{2}$ obtained from an expansion of pyridine in $\mathrm{He}$ without water. In this case the $\mathrm{PyH}^{+}$ spectrum is similar to the $\mathrm{Py}^{+}$spectrum. (a)

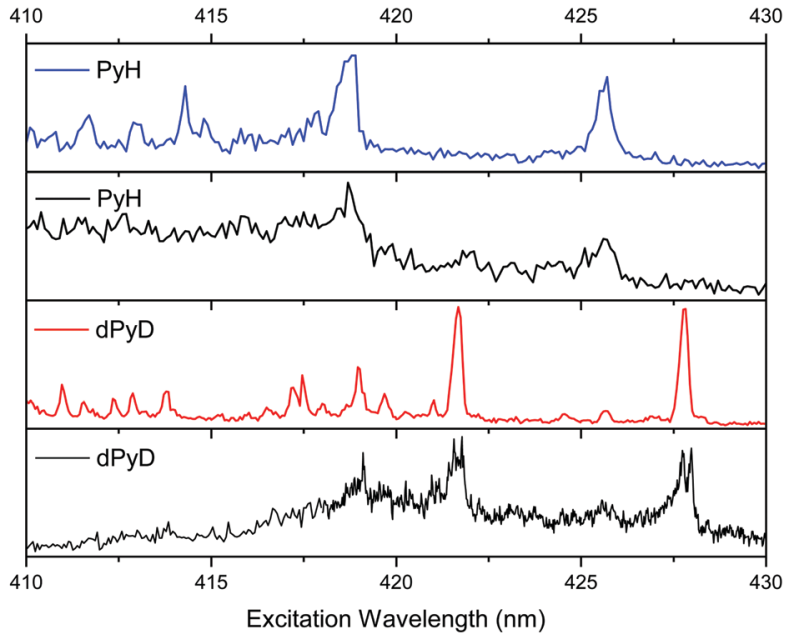

Fig. 5 Two-photon ionization spectra, comparison between: (a) $\mathrm{PyH}^{\bullet}$ issued from the discharge source, (b) $\mathrm{PyH}^{\bullet}$ from the reaction of Py- $\left(\mathrm{H}_{2} \mathrm{O}\right)_{n}$ excited at $255 \mathrm{~nm}$, (c) dPyD from the discharge source, (d) dPyD from the reaction of the $\mathrm{dPy}-\left(\mathrm{D}_{2} \mathrm{O}\right)_{n}$ clusters excited at $255 \mathrm{~nm}$.

pyridinyl radicals produced in the discharge source are ionized (Fig. 4a). Thus, there is a clear and unambiguous signature of the processes involved in the experiment. In pure pyridine clusters, there is a ionic reaction leading to $\mathrm{PyH}^{+}$, while in expansion containing water, excited pyridine-water clusters react to produce the pyridinyl radical, clearly identified by its ionization threshold. The water splitting reaction is then clearly demonstrated.

Another method to detect the $\mathrm{PyH}^{\bullet}$ radical is through the two-photon ionization scheme in the visible spectral region. In Fig. 5 are presented the pump probe $\mathrm{PyH}^{\bullet}$ and $\mathrm{dPyD}^{\bullet}$ (fully deuterated pyridinyl) action spectra in comparison with the spectra obtained for the cold species issued from the discharge source. This unambiguously ascertains that the $\mathrm{PyH}^{+}$signal detected with the probe laser is due to the ionization of the $\mathrm{PyH}^{\bullet}$ radical. It can be noticed that the action spectra resulting from the reaction in clusters are broadened as compared to the spectra of the cold species produced in the discharge source, which implies that the $\mathrm{PyH}^{\bullet}$ or $\mathrm{dPyD}^{\bullet}$ reaction products are produced with some internal energy. Note that the $\mathrm{dPyD}^{\bullet}$ vibronic bands are red-shifted as compared to the $\mathrm{PyH}^{\bullet}$ bands because the heavier deuterium mass induces an isotopic shift reducing the vibrational frequencies.

\section{For which cluster size does the reaction occur?}

We could expect to identify the parent ion from the action spectrum recorded in probing $\mathrm{PyH}^{\bullet}$ while scanning the pump laser. The $\mathrm{PyH}^{\bullet}$ action spectrum is compared to the R2PI spectrum of the Py- $\left(\mathrm{H}_{2} \mathrm{O}\right)$ or $(\mathrm{Py})_{2}$ complex in Fig. S4 (ESI $\left.\dagger\right)$. As for the free molecule the spectra are broad and not well resolved making the identification of the parent molecule difficult, even though the $\mathrm{PyH}^{\bullet}$ action spectrum and the $\mathrm{Py}-\left(\mathrm{H}_{2} \mathrm{O}\right)$ R2PI spectra are similar.

Since we can record the one-color two-photon absorption/ ionization spectrum at the mass of the $\mathrm{Py}-\mathrm{H}_{2} \mathrm{O}$ complex, the 
complex does not react very quickly, otherwise it could not absorb the second photon necessary to ionize. In fact, there is a kind of contradiction to use spectroscopy to determine the reacting cluster size since, if the cluster reacts quickly, it will not be ionized thus not observed. Along these lines, it can be noted that the Py- $\left(\mathrm{H}_{2} \mathrm{O}\right)_{2}$ complex stays very weak and the $\mathrm{Py}-\left(\mathrm{H}_{2} \mathrm{O}\right)_{3}$ cluster is barely observed. However, the similarity between the $\mathrm{PyH}^{\bullet}$ and $\mathrm{Py}-\mathrm{H}_{2} \mathrm{O}$ spectra, shown in Fig. S4 (ESI $\dagger$ ), indicates that pyridine-water clusters are responsible for the reaction.

The only way to get some idea of the clusters responsible for the reaction is by changing expansion conditions.

The pressure effect measurements shown in Fig. 6 have been recorded to see if we could correlate the $\mathrm{PyH}^{\bullet}$ signal with some $\mathrm{Py}-\left(\mathrm{H}_{2} \mathrm{O}\right)_{n}$ cluster sizes since the mean cluster size increases with the backing pressure.

The variation of the population of a given cluster size with the backing pressure should follow a Poisson distribution. To calibrate the signal versus the Poisson distribution, we assume that the Py- $\mathrm{H}_{2} \mathrm{O}$ complex follows a second order Poisson distribution $P(\lambda=2)$, and fitted the observed signal with this distribution (Fig. 6 left upper panel). As can be seen, the Py- $\left(\mathrm{H}_{2} \mathrm{O}\right)_{2}$ complex follows a $P(\lambda=3)$ distribution, which provides some confidence in the approach. The $\mathrm{PyH}^{\bullet}$ signal (lower right panel) clearly does not follow the same variation of the signal as the
1-1 or 1-2 complexes but can be fitted with a sum of distributions with $P(\lambda \geq 4)$ to be reproduced. At the same time we did not observe $\mathrm{Py}-\left(\mathrm{H}_{2} \mathrm{O}\right)_{n}$ clusters with $n>3$, which probably means that these clusters react faster. This situation is in line with the experiments concerning pyridine-water anions, where it is suggested that with $n>3$, only structures exhibiting proton transfer $\left[\mathrm{PyH}^{\bullet} \mathrm{OH}\right]^{-}\left(\mathrm{H}_{2} \mathrm{O}\right)_{n-1}$ are present, best described as $\left(\mathrm{PyH}^{\bullet} \mathrm{OH}^{-}\right)\left(\mathrm{H}_{2} \mathrm{O}\right)_{n-1} \cdot{ }^{5,6}$

Why are large $(n \geq 4)$ clusters necessary to induce the reaction? The 1-1 complex is observed though a one-color two-photon ionization scheme, which implies a relatively long excited state lifetime i.e. a barrier to the reaction: in the absence of a barrier the reaction rate would be very fast, the excited state lifetime very short and the complex would not be ionized. The simplest explanation for the reactivity of larger clusters would be the lowering of the barrier as the cluster size increases. This effect is well known in the case of ionic reactions in which the transition state is stabilized by solvent molecules. But we are dealing with a radical reaction, which is a lot less sensitive to the solvent effect. As a matter of fact Reimers et al. calculated that the barrier is not strongly influenced by the solvent, ${ }^{1}$ so that this effect should be marginal.

A second hypothesis can be suggested. Ab initio calculations show that the crossing between the ground and the reacting
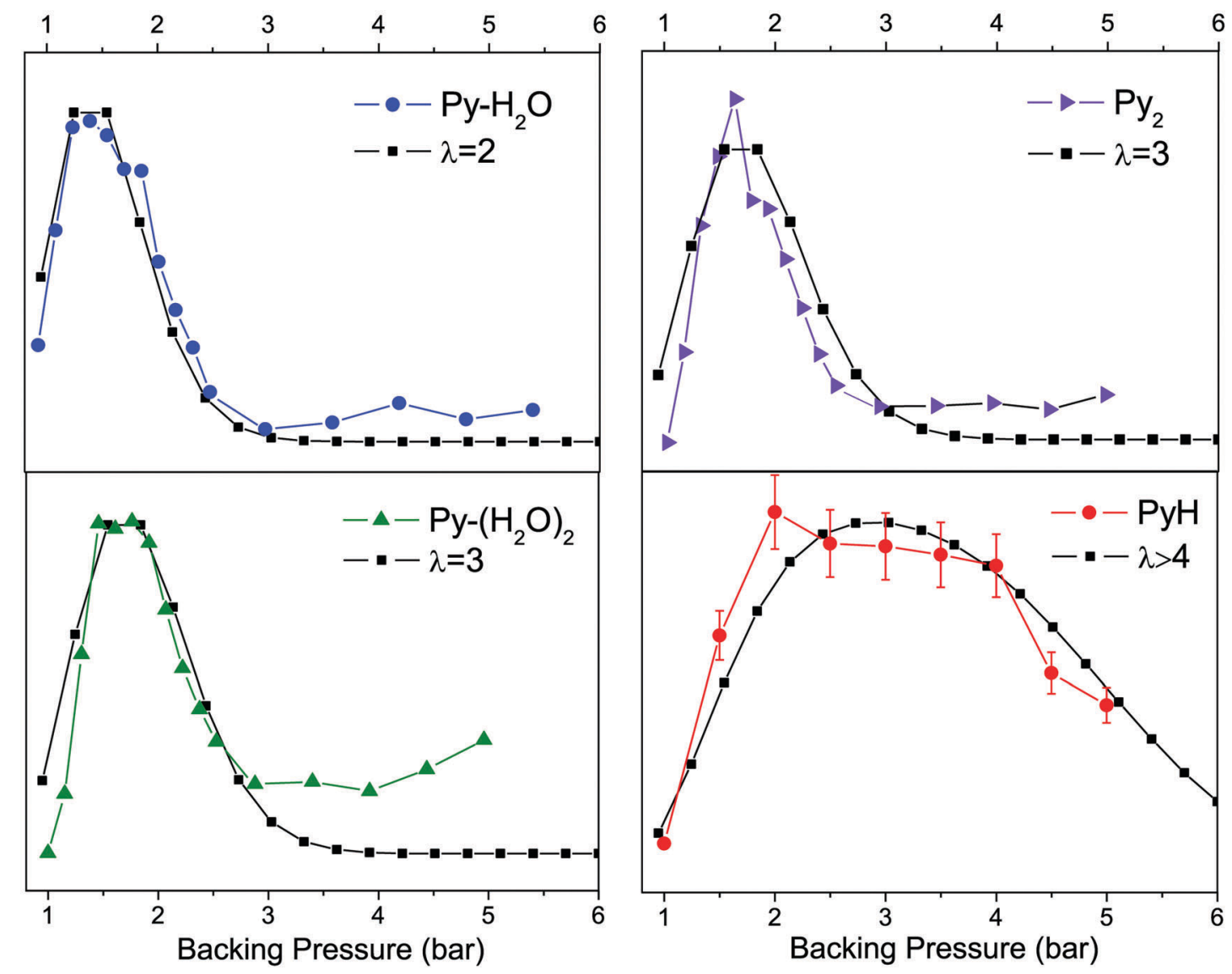

Fig. 6 Variation of the ion intensities as a function of the backing pressure. When the pressure increases the cluster size increases. In red the PyH ${ }^{\bullet}$ signal seems to indicate that large clusters are involved in the reaction. 


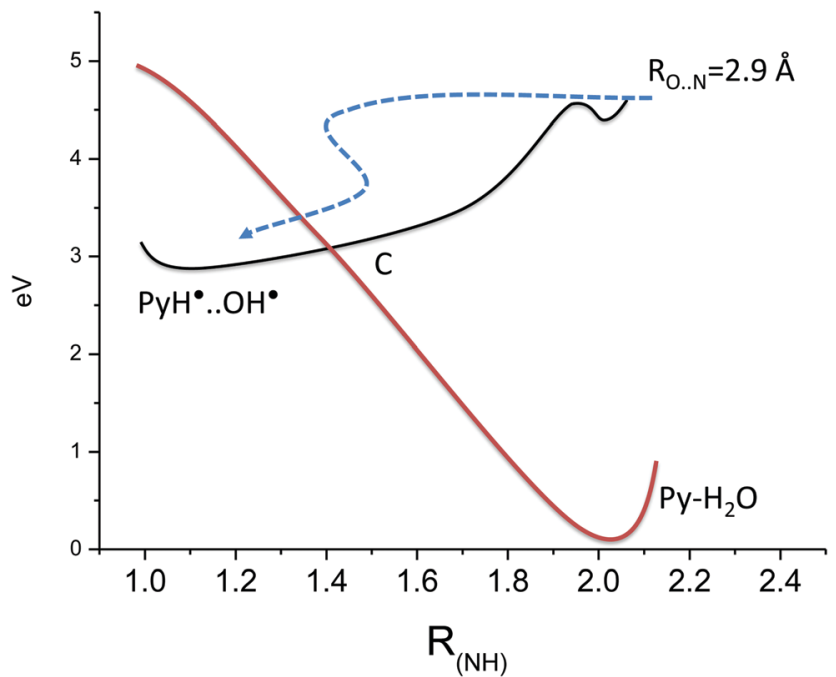

Fig. 7 Schematic representation of the reaction mechanism. There is a competition between the stabilization of $\mathrm{PyH}^{\bullet}$ and the recombination in $\mathrm{Py}-\mathrm{H}_{2} \mathrm{O}$, which depends on how fast the excess energy is released and how fast the $\mathrm{OH}^{\bullet}$ radical is leaving the cluster. The dotted blue arrow represents the schematic reaction pathway showing the efficient energy relaxation induced by the solvent molecules, which prevents multiple passages over the conical intersection $\mathrm{C}$. The potential curves are adapted from ref. 2.

excited state occurs at an $\mathrm{OH}$ distance of 1.9/1.8 $\AA$, i.e. for $\mathrm{NH}$ around 1.1/1.2 $\AA$ if the NO distance is fixed, i.e. if the $\mathrm{OH}$ fragment did not move during the $\mathrm{H}$ transfer. As seen in Fig. 7, the excited state correlates with the $\mathrm{PyH}^{\bullet}+\mathrm{OH}^{\bullet}$ ground state. Due to the $\mathrm{H} / \mathrm{OH}$ mass ratio, the oxygen atom does not move very quickly as compared to the $\mathrm{H}$ atom. In order to be trapped in the weak $\mathrm{PyH}^{\bullet}$ potential well, the excess energy has to be removed quite efficiently and the reaction path represented by the dotted blue arrow will cross only once the conical intersection leading back to the initial $\mathrm{Py}-\mathrm{H}_{2} \mathrm{O}$ system. This is probably the role of the other water molecules in the cluster. In the absence of such a dissipating bath the $\mathrm{H}$ atom will bounce on the $\mathrm{N}$ atom and recombine in the most stable $\mathrm{Py}-\mathrm{H}_{2} \mathrm{O}$ form through the conical intersection (C), and further dissociate into $\mathrm{Py}+\mathrm{H}_{2} \mathrm{O}$.

In summary of this part, the $\mathrm{PyH}^{\bullet}$ photo-product does not seem to come from the 1-1 complex and the fact that the 1-1 complex can be ionized means that its excited state lifetime is relatively long (longer than a few ps,...). It seems that larger clusters are necessary, probably because they react more efficiently and because the surrounding water molecules can dissipate the excess energy necessary to trap the $\mathrm{PyH}^{\bullet}$ product.

\section{Photodissociation of $\mathrm{PyH}^{\bullet}$}

In order to use pyridine as a photo-catalyst, one should ensure that the absorption of a photon by $\mathrm{PyH}^{\bullet}$ can lead to $\mathrm{H}$ loss and recovery of the catalyst. Calculations ${ }^{2}$ indicate that there is a $\pi \sigma^{*}$ state quasi dissociative along the $\mathrm{NH}$ coordinate, which crosses the $\pi \pi^{*}$ excited state and leads to $\mathrm{H}$ atom loss. The barrier seems to be quite low, the excitation of the first observed transition at $425.6 \mathrm{~nm}(2.91 \mathrm{eV})$ is above the dissociation limit in
Py $+\mathrm{H}(1.5 \mathrm{eV})$, thus $\mathrm{PyH}^{\bullet}$ photodissociation is energetically allowed.

In the first step, we tried to evidence this process in exciting cold $\mathrm{PyH}^{\bullet}$ issued from the discharge source. The detection scheme requires the discharge to produce $\mathrm{PyH}^{\bullet}$, one photon in the $420 \mathrm{~nm}$ range to excite $\mathrm{PyH}^{\bullet}$ and a probe photon at $255 \mathrm{~nm}$ to ionize Py by resonant one-color two-photon absorption. The result was disappointing and no Py could be evidenced in this experiment.

In the second step, we studied the dissociation process from hot $\mathrm{PyH}^{\bullet}$ issued from the reaction in the clusters. In this case, the first laser at $255 \mathrm{~nm}$ excites the clusters, and the second powerful laser in the visible region is used to excite and ionize $\mathrm{PyH}^{\bullet}$ (via $\mathrm{R} 2 \mathrm{PI}$ ) and to ionize Py through a four-photon absorption process. The results are presented in Fig. 8.

The first set of data concerning the hydrogenated system is the richest (Fig. 8a). When $\mathrm{PyH}^{\bullet}$ is produced by reaction in $\mathrm{Py}-\left(\mathrm{H}_{2} \mathrm{O}\right)_{n}$ clusters, the first band at $23500 \mathrm{~cm}^{-1}$ is observed only in the $\mathrm{PyH}^{\bullet}$ channel and not in the Py channel. At $23600 \mathrm{~cm}^{-1} \mathrm{a}$ threshold is observed in the Py channel (arrow in the upper panel of Fig. 8a) and the second band at $23880 \mathrm{~cm}^{-1}$ appears in both Py and $\mathrm{PyH}^{\bullet}$ channels. A third vibrational band is observed very weakly in the cold $\mathrm{PyH}^{\bullet}$ spectrum (from the discharge) and very strongly in the Py channel. In the case of the deuterated species, no structure is observed in the Py channel, only a broad band, which is also observed in the $\mathrm{dPyD}^{\bullet}$ channel underneath the vibronic bands.

These data can be rationalized in the following way: the dissociative $\pi \sigma^{*}$ state has a different symmetry than the optically active state $\pi \pi^{*}$. Thus, out of plane vibrations or high rotational excitations are necessary to induce a coupling between these states. In the cold $\mathrm{PyH}^{\bullet}$ from the discharge, the rotational excitation is low and the out of plane vibrations may not be very active, so that no dissociation is observed. The out of plane vibrations could be populated by the vibrational redistribution but in such a small cyclic molecule with a relatively small vibrational energy excess, ${ }^{39,40}$ this process should not be competitive with the radiative process.

For the hydrogenated species, the fragmentation threshold is clearly observed at $23600 \mathrm{~cm}^{-1}$ and the observation of a common vibrational band at $23880 \mathrm{~cm}^{-1}$ in all the spectra is good evidence that the Py observed comes from the excitation of $\mathrm{PyH}^{\bullet}$. An alternative explanation could have been the excitation of the clusters at $255 \mathrm{~nm}$, relaxation toward the Py triplet state and evaporation of water molecules, followed by the ionization of the triplet by the probe laser. Since the probe laser is delayed in time by $600 \mathrm{~ns}$ and since it seems that the Py triplet is relatively short lived (72 $\mathrm{ns}),{ }^{1,41}$ this mechanism will not match our measurements.

The third band observed at $24300 \mathrm{~cm}^{-1}$ in the Py channel might be assigned to some out of plane vibrations, which is weakly seen on the spectrum of the cold radical but will enhance strongly the coupling between the $\pi \pi^{*}$ and the $\pi \sigma^{*}$ states and thus increase the fragmentation efficiency.

The contrast with the deuterated system is quite impressive, no structure is observed in the Py fragmentation pathway 
(a)

(Fig. 8b). The H/D loss is probably controlled by some tunneling mechanism, and the deuterated species will tunnel less efficiently, or need a higher internal energy to cross efficiently the barrier as compared to the hydrogenated species. In this case, only very hot $\mathrm{dPyD}$ molecules can dissociate. As already stated, the Py channel detection occurs via a four-photon ionization process, which may induce artifacts in the detection scheme (accidental resonances). Detection using a third laser to ionize Py with two-photon absorption would be worthwhile.

\section{Conclusions}

The spectroscopy of the pyridinyl radical has been characterized at low temperature in a supersonic expansion and its ionization potential has been determined. Since $\mathrm{PyH}^{\bullet}$ can be probed through one photon ionization in the UV, pump probe experiments have demonstrated that the UV excitation of $\mathrm{Py}-\left(\mathrm{H}_{2} \mathrm{O}\right)_{n}$ clusters leads to the formation of a pyridinyl radical, thus we have evidenced the water splitting reaction using pyridine as a photo-sensitizer. We have also evidenced the role of the surrounding molecules, some extra water molecules in the clusters being necessary to stabilize detecting $\mathrm{dPy}^{+}$. (b)

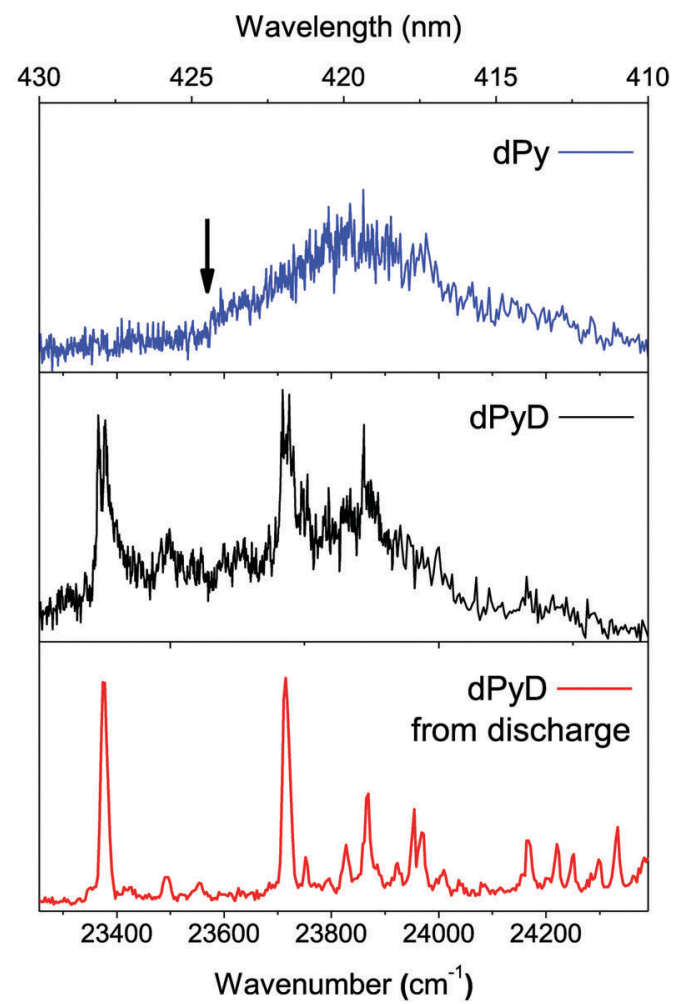

Fig. 8 (a) Hydrogenated pyridine: lower panel, the two-photon excitation/ionization spectrum of cold PyH• produced in the discharge; middle panel, at $255 \mathrm{~nm}$ and detecting $\mathrm{dPyD}^{+}$by R2Pl; upper panel, the action spectrum of $\mathrm{dPyD}^{\bullet}$ produced after the excitation of $\mathrm{dPy}-\left(\mathrm{D}_{2} \mathrm{O}\right)_{n}$ clusters and

the $\mathrm{PyH}^{\bullet}$ product. It would probably be quite instructive to detect the other product of the reaction, the $\mathrm{OH}^{\bullet}$ radical.

More work has to be done to fully characterize this reaction, in particular the cluster sizes that lead to the reaction may be studied through infrared hole burning experiments, and the dynamics of the excited states may be investigated in the picosecond regime.

The last step of the process, i.e. the regeneration of the catalyst through the photo-detachment of the hydrogen atom $\left(\mathrm{PyH}^{\bullet}+h \nu \rightarrow \mathrm{Py}+\mathrm{H}\right)$ has been studied. It seems that only hot $\mathrm{PyH}^{\bullet}$ radicals can lose hydrogen atoms. Other aromatic molecules ${ }^{42,43}$ are expected to lead to similar water splitting reactions and will be tested in the near future.

\section{Acknowledgements}

We are highly indebted to Pr A. L. Sobolewski and W. Domcke for triggering our interest to this reaction mechanism and for very helpful discussions. We acknowledge the use of the computing facility cluster GMPCS of the LUMAT federation (FR LUMAT 2764). 


\section{References}

1 J. R. Reimers and Z.-L. Cai, Phys. Chem. Chem. Phys., 2012, 14, 8791-8802.

2 X. Liu, A. L. Sobolewski, R. Borrelli and W. Domcke, Phys. Chem. Chem. Phys., 2013, 15, 5957.

3 X. Liu, A. L. Sobolewski and W. Domcke, J. Phys. Chem. A, 2014, 118, 7788-7795.

4 F. Riboni, E. Selli and A. J. Colussi, J. Phys. Chem. A, 2015, 119, 4433-4438.

5 A. F. DeBlase, C. T. Wolke, G. H. Weddle, K. A. Archer, K. D. Jordan, J. T. Kelly, G. S. Tschumper, N. I. Hammer and M. A. Johnson, J. Chem. Phys., 2015, 143, 144305.

6 Y. Wang, X. Zhang, S. Lyapustina, M. M. Nilles, S. Xu, J. D. Graham, K. H. Bowen, J. T. Kelly, G. S. Tschumper and N. I. Hammer, Phys. Chem. Chem. Phys., 2016, 18, 704-712.

7 A. Müller, F. Talbot and S. Leutwyler, J. Am. Chem. Soc., 2002, 124, 14486-14494.

8 T. Droz, R. Knochenmuss and S. Leutwyler, J. Chem. Phys., 1990, 93, 4520.

9 K. Sakota, C. Okabe, N. Nishi and H. Sekiya, J. Phys. Chem. A, 2005, 109, 5245-5247.

10 G. A. Pino, I. Alata, C. Dedonder, C. Jouvet, K. Sakota and H. Sekiya, Phys. Chem. Chem. Phys., 2011, 13, 6325-6331.

11 C. Dedonder-Lardeux, D. Grosswasser, C. Jouvet and S. Martrenchard, PhysChemComm, 2001, 4, 21-23.

12 G. A. Pino, C. Dedonder-Lardeux, G. Grégoire, C. Jouvet, S. Martrenchard and D. Solgadi, J. Chem. Phys., 1999, 111, 10747.

13 G. Grégoire, C. Dedonder-Lardeux, C. Jouvet, S. Martrenchard, A. Peremans and D. Solgadi, J. Phys. Chem. A, 2000, 104, 9087-9090.

14 O. David, C. Dedonder-Lardeux, C. Jouvet and A. L. Sobolewski, J. Phys. Chem. A, 2006, 110, 9383-9387.

15 M. Miyazaki, R. Ohara, K. Daigoku, K. Hashimoto, J. R. Woodward, C. Dedonder, C. Jouvet and M. Fujii, Angew. Chem., Int. Ed., 2015, 54, 15089-15093.

16 N. Tsuji, S.-I. Ishiuchi, C. Jouvet, C. Dedonder-Lardeux, M. Miyazaki, M. Sakai and M. Fujii, ChemPhysChem, 2011, 12, 1928-1934.

17 G. A. Pino, A. N. Oldani, E. Marceca, M. Fujii, S. Ishiuchi, M. Miyazaki, M. Broquier, C. Dedonder and C. Jouvet, J. Chem. Phys., 2010, 133, 124313.

18 A. Carrera, I. B. Nielsen, P. Carcabal, C. Dedonder, M. Broquier, C. Jouvet, W. Domcke and A. L. Sobolewski, J. Phys. Chem. A, 2009, 130, 024302.

19 V. Stert, L. Hesse, H. Lippert, C. P. Schulz and W. Radloff, J. Phys. Chem. A, 2002, 106, 5051-5053.
20 S. Ishiuchi, K. Daigoku, M. Saeki, M. Sakai, K. Hashimoto and M. Fujii, J. Chem. Phys., 2002, 117, 7077.

21 S. Ishiuchi, M. Sakai, K. Daigoku and T. Ueda, Chem. Phys. Lett., 2001, 347, 87-92.

22 S.-I. Ishiuchi, K. Daigoku, K. Hashimoto and M. Fujii, J. Chem. Phys., 2004, 120, 3215-3220.

23 O. David, C. Dedonder-Lardeux, C. Jouvet, H. Kang, S. Martrenchard, T. Ebata and A. L. Sobolewski, J. Chem. Phys., 2004, 120, 10101.

24 A. Courty, M. Mons and I. Dimicoli, J. Phys. Chem. A, 1998, 102, 6590-6600.

25 M. Miyazaki, A. Takeda, S. Ishiuchi, M. Sakai, O. Dopfer and M. Fujii, Phys. Chem. Chem. Phys., 2011, 13, 2744-2747.

26 K. Tanabe, M. Miyazaki, M. Schmies, A. Patzer, M. Schütz, H. Sekiya, M. Sakai, O. Dopfer and M. Fujii, Angew. Chem., Int. Ed., 2012, 51, 6604-6607.

27 H. M. Kim, K. Y. Han, J. Park, G.-S. Kim and S. K. Kim, J. Chem. Phys., 2008, 128, 041104.

28 C. Jouvet and B. Soep, Chem. Phys. Lett., 1983, 96, 426-428.

29 W. H. Breckenridge, C. Jouvet and B. Soep, J. Chem. Phys., 1986, 84, 1443.

30 M. Boivineau, J. Le Calve, M. C. Castex and C. Jouvet, Chem. Phys. Lett., 1986, 130, 208.

31 M. Dantus, M. J. Rosker and A. H. Zewail, J. Chem. Phys., 1987, 87, 2395-2397.

32 A. Zehnacker-Rentien and M. A. Suhm, Angew. Chem., Int. Ed., 2008, 47, 6970-6992.

33 J. Wanna, J. A. Menapace and E. R. Bernstein, J. Chem. Phys., 1986, 85, 1795-1805.

34 C. Jouvet, C. Lardeux-Dedonder, M. Richard-Viard, D. Solgadi and A. Tramer, J. Phys. Chem. A, 1990, 94, 5041-5048.

35 C. Dedonder-Lardeux, C. Jouvet, M. Richard-Viard and D. Solgadi, J. Chem. Phys., 1990, 91, 2828.

36 I. Alata, R. Omidyan, M. Broquier, C. Dedonder and C. Jouvet, Chem. Phys., 2012, 399, 224-231.

37 TURBOMOLE V6.3 2011, a Dev. Univ. Karlsruhe Forschungszentrum Karlsruhe GmbH, 1989-2007, TURBOMOLE GmbH, since 2007; available from http//www.turbomole.com.

38 M. Riese, Z. Altug and J. Grotemeyer, Phys. Chem. Chem. Phys., 2006, 8, 4441-4448.

39 J. B. Hopkins, D. E. Powers and R. E. Smalley, J. Chem. Phys., 1980, 73, 683-687.

40 R. E. Smalley, Annu. Rev. Phys. Chem., 1983, 34, 129.

41 K. Enomoto, J. A. LaVerne, S. Seki and S. Tagawa, J. Phys. Chem. A, 2006, 110, 9874-9879.

42 X. Liu, T. N. V. Karsili, A. L. Sobolewski and W. Domcke, J. Phys. Chem. B, 2015, 119, 10664-10672.

43 T. N. V. Karsili, D. Tuna, J. Ehrmaier and W. Domcke, Phys. Chem. Chem. Phys., 2015, 17, 32183-32193. 